\title{
Discrete and Canonical Quantum Variables
}

\author{
M. Ruzzi, S.S.L. Machado, \\ Departamento de Física, UFSC, 88040-900, Florianópolis, SC, Brazil \\ and D. Galetti \\ Instituto de Física Teórica, UNESP, 01405-900, São Paulo, SP, Brazil
}

Received on 30 October, 2002

A connection betweend discrete, Cartesian and angular quantum variables is obtained and discussed.

\section{Introduction - Schwinger's approach}

Quantum mechanics, and in particular the language of quantum mechanics, is deeply entangled with the way physics is done on its many branches. Therefore, the interpretation of the formalism might have a crucial role on areas where both theoretical and experimental efforts are highly developed. Nuclear physics, with its need to take into account so many different effects - which are better understood mostly in languages of different theories - is a sound example of the above remark. Motivated by this basic idea, we shall here explore some aspects of the formal structure of quantum mechanics, in a commented overview of the work recently published on $[1,2]$.

In the beginning of the sixties Schwinger had developed a program of treating quantum degrees of freedom characterized by a finite number of states, therefore with no classical counterpart. We start considering quantum systems described on Hilbert spaces of finite dimension. Let the set $\left\{\left|u_{k}\right\rangle\right\}$ denote the eigenstates of an arbitrary Hermitian operator acting on the space of interest. So, the states $\left\{\left|u_{k}\right\rangle\right\}$ might represent a multiplet in a closed shell, eigenstates of the quantized axis of spin, isospin, and so on. The index $k$ is an integer which runs from 0 to $N-1$.

We define an operator $V$ as:

$$
V=\sum_{k=0}^{N-1}\left|u_{k-1}\right\rangle\left\langle u_{k}\right|
$$

Where we use the notation convention $\left|u_{j}\right\rangle \equiv\left|u_{j(\bmod N)}\right\rangle$. Two integers $a$ e $b$ are said to be congruent modulo $m$ when $m$ divides the difference $a-b$ to a integer $l$, so $a=b+m l$, denoted $a \equiv b(\bmod m)$. For equation $(1)$, in particular, we use that $N-1 \equiv-1(\bmod N)$.

The reasoning behind this notation is plain simple: initially, only kets denoted by $\left\{\left|u_{0}\right\rangle,\left|u_{1}\right\rangle \ldots\left|u_{N-1}\right\rangle\right\}$ have any kind of meaning. A ket labeled by $\left|u_{N}\right\rangle$, for example, does not refer to any state of the initial basis, or to any state in particular at all. The modulo $N$ extraction convention on the indices, however, gives meaning to this label, and to any other out of the first determination $[0, N-1]$, as $\left|u_{N(\bmod N)}\right\rangle \equiv\left|u_{0}\right\rangle,\left|u_{N+1(\bmod N)}\right\rangle \equiv\left|u_{1}\right\rangle$, and so on.

Applying $V^{s}$, where $s$ is an integer, on a given $\left|u_{j}\right\rangle$,

$$
V^{s}\left|u_{j}\right\rangle=\left|u_{j-s}\right\rangle,
$$

and we see that the action of integer powers of $V$ shifts the index of the state, and in particular, on $\left|u_{0}\right\rangle$ it results on $\left|u_{N-1}\right\rangle$, 'connecting' the extremes of the set. Choosing $s=N$ above and using the fact that $j-N=j(\bmod N)$ we conclude that $V$ is a cyclic operator, that is, $V^{N}=\hat{1}$.

Let us look for the eigenstates (and associated eigenvalues) of the operator $V$,

$$
V\left|v_{j}\right\rangle=v_{j}\left|v_{j}\right\rangle
$$

From $V^{N}=\hat{1}$ we see that $\left(v_{j}\right)^{N}=1$, then

$$
v_{j}=\exp \left[\frac{2 \pi i}{N} j\right] .
$$

For the eigenstates of $V$ we obtain, once we decompose $\left|v_{j}\right\rangle$ in the $\left\{\left|u_{k}\right\rangle\right\}$ basis,

$$
\left\langle u_{l} \mid v_{j}\right\rangle=\frac{1}{\sqrt{N}} \exp \left[\frac{2 \pi i}{N} j l\right],
$$

where $\frac{1}{\sqrt{N}}$ is an assumed real normalization factor.

We can now construct, similarly to $V$, an operator acting on $\left\{\left|v_{j}\right\rangle\right\}$ as

$$
U\left|v_{j}\right\rangle=\left|v_{j+s}\right\rangle
$$

where modulo $N$ notation is still assumed. As $V$ lowers the index of the state $\left|u_{j}\right\rangle, U$ raises the index of $\left|v_{j}\right\rangle$. Choosing the integer $s=N$ we see that also $U^{N}=\hat{1}$. Finally, we can see that our initial states are precisely the eigenstates of $U$, 


$$
U\left|u_{j}\right\rangle=u_{j}\left|u_{j}\right\rangle .
$$

whose eigenvalues are also $N$-th roots of unity,

$$
u_{j}=\exp \left[\frac{2 \pi i}{N} j\right]
$$

As we saw, both sets are connected by a discrete Fourier transform, presenting a maximum degree of incompatibility. Finally, it can be easily seen that the pair $(U, V)$ obeys Weyl algebra

$$
U^{j} V^{l}=\exp \left[\frac{2 \pi i}{N} j l\right] V^{l} U^{j} .
$$

\section{The limit to the continuum}

\section{II.1 Cartesian Variables}

Lets us first introduce a scaling factor

$$
\epsilon=\sqrt{\frac{2 \pi}{N}},
$$

which will become infinitesimal as $N \rightarrow \infty$. Then, two Hermitian operators $\{P, Q\}$, (for simplicity, odd $N^{\prime}$ s will be considered),

$$
\begin{gathered}
P=\sum_{j=-\frac{N-1}{2}}^{\frac{N-1}{2}} j \epsilon^{\delta} p_{0}\left|v_{j}\right\rangle\left\langle v_{j}\right| \\
Q=\sum_{j^{\prime}=-\frac{N-1}{2}}^{\frac{N-1}{2}} j^{\prime} \epsilon^{2-\delta} q_{0}\left|u_{j^{\prime}}\right\rangle\left\langle u_{j^{\prime}}\right|,
\end{gathered}
$$

constructed out of the projectors of the eigenstates of $U$ and $V . \delta$ is a free parameter on the open interval $(0,2),\left\{p_{0}, q_{0}\right\}$ are real parameters carrying units of momentum and position, respectively, and $\epsilon^{\delta} p_{0}$ and $\epsilon^{2-\delta} q_{0}$ are then the distance between successive eigenvalues of the $P$ and $Q$ operators. With the help of these, we can rewrite the $U$ and $V$ operators as

$$
V=\exp \left[\frac{i \epsilon^{2-\delta} P}{p_{0}}\right] \quad U=\exp \left[\frac{i \epsilon^{\delta} Q}{q_{0}}\right] .
$$

Let also both eigenstate sets be relabeled as

$$
\left|v_{j}\right\rangle \equiv|p\rangle \quad\left|u_{j^{\prime}}\right\rangle=|q\rangle ; \quad q=q_{0} \epsilon^{2-\delta} j^{\prime} \quad p=p_{0} \epsilon^{\delta} j .
$$

With that,

$$
P=\sum_{p=-\frac{N-1}{2} \epsilon^{\delta} p_{0}}^{\frac{N-1}{2} \epsilon^{\delta} p_{0}} p|p\rangle\left\langle p\left|\quad Q=\sum_{q=-\frac{N-1}{2} \epsilon^{2-\delta} q_{0}}^{\frac{N-1}{2} \epsilon^{2-\delta} q_{0}} q\right| q\right\rangle\langle q|,
$$

and Eqs. (6) and (2) now will read

$\exp \left[\frac{i p^{\prime} Q}{p_{0} q_{0}}\right]|p\rangle=\left|p+p^{\prime}\right\rangle \quad \exp \left[\frac{i q^{\prime} P}{p_{0} q_{0}}\right]|q\rangle=\left|q-q^{\prime}\right\rangle$.

if $\left\{p^{\prime}, q^{\prime}\right\}$ are defined following the recipe of (13). One must not forget that the new variables are still bounded by the modulo $N$ notation, that is $q=q\left(\bmod N q_{0} \epsilon^{2-\delta}\right)$ and $p=p\left(\bmod N q_{0} \epsilon^{\delta}\right)$, to put it roughly.

The $N \rightarrow \infty$ limit can now be easily performed. For $\delta$ assuming any value in the open interval $(0,2)$, both Hermitian operators on Eqs.(14) will feature an unbounded and continuous spectrum, as the limit leads them to:

$$
P=\int_{-\infty}^{\infty} p|p\rangle\left\langle p\left|d p \quad Q=\int_{-\infty}^{\infty} q\right| q\right\rangle\langle q| d q
$$

Eqs. (15) now are valid for any real numbers $\left\{p, q, p^{\prime}, q^{\prime}\right\}$. It must be observed that, in the way they are obtained, the labels $\{p, q\}$ span the set of all rational numbers (times a given irrational), which is a proper subset of the set of real numbers. On the other hand, every real number can be written as the limit of an infinite sequence of rational numbers. Then the expression

$\exp \left[\frac{i\left(p^{\prime}+p^{\prime \prime}+p^{\prime \prime \prime}+\ldots\right) Q}{p_{0} q_{0}}\right]|p\rangle=\left|p+p^{\prime}+p^{\prime \prime}+p^{\prime \prime \prime}+\ldots\right\rangle$

might converge to any real eigenvalue and its associated eigenvector. This is enough to ensure that the whole usual Hilbert space of usual canonical variables is recovered[5]. Also, after the limit is performed the modulo $N$ notation becomes irrelevant, and the familiar relations are easily recovered from their discrete counterparts

$$
\begin{gathered}
Q|q\rangle=q|q\rangle, \quad\left\langle q^{\prime} \mid q\right\rangle=\delta\left(q^{\prime}-q\right), \quad-\infty \leq q^{\prime}, q \leq \infty \\
P|p\rangle=p|p\rangle, \quad\left\langle p^{\prime} \mid p\right\rangle=\delta\left(p^{\prime}-p\right), \quad\langle p \mid q\rangle=\frac{1}{\sqrt{2 \pi p_{0} q_{0}}} \exp \left(\frac{i p q}{p_{0} q_{0}}\right) .
\end{gathered}
$$


Therefore the results for a degree of freedom endowed with a usual position-momentum canonical pair of variables are completely reproduced, provided that the product of the parameters $p_{0} q_{0}$ is set to $\hbar$.

The matrix elements (still in the discrete case) of the $P$ operator in the $Q$ representation are easily seen to be

$$
\left\langle q|P| q^{\prime}\right\rangle=\frac{1}{N} \sum_{p=-\frac{N-1}{2} \epsilon^{\delta} p_{0}}^{\frac{N-1}{2} \epsilon^{\delta} p_{0}} p \exp \left(\frac{i p\left(q^{\prime}-q\right)}{p_{0} q_{0}}\right),
$$

leading to the well known result for the continuum case,

$$
\left\langle q|P| q^{\prime}\right\rangle=\frac{1}{2 \pi p_{0} q_{0}} \int_{-\infty}^{\infty} d p p \exp \left(\frac{i p\left(q^{\prime}-q\right)}{p_{0} q_{0}}\right) .
$$

Is is interesting to see that the r.h.s. of Eq.(21) is a representation of the first derivative of the Dirac delta, but its discrete counterpart cannot be directly associated to a finite difference operator ${ }^{1}$.

As the results above does not depend on $\delta$ (once it is on the interval $(0,2)$, so that the resulting integrals cover all space), we see that the cartesian coordinates are overdetermined by the limiting process.

\section{II.2 Angular Variables}

The limiting procedure changes dramaticaly if we consider the extreme situation $\delta=0$ (or $\delta=2$, which is equivalent). In this case one of the variables is not scaled at all and what follows is almost identical to the well known PeggBarnett[3] scheme for quantum optics (here, for simplicity, the reference angle is set to zero). One would have

$$
V=\exp \left[\frac{i \epsilon^{2} M}{m_{0}}\right] \quad U=\exp \left[\frac{i \Theta}{\theta_{0}}\right]
$$

where

$$
\begin{gathered}
M=\sum_{j=-\frac{N-1}{2}}^{\frac{N-1}{2}} j m_{0}\left|v_{j}\right\rangle\left\langle v_{j}\right| \\
\Theta=\sum_{j^{\prime}=-\frac{N-1}{2}}^{\frac{N-1}{2}} \epsilon^{2} j^{\prime} \theta_{0}\left|u_{j^{\prime}}\right\rangle\left\langle u_{j^{\prime}}\right| .
\end{gathered}
$$

The pair $\left\{m_{0}, \theta_{0}\right\}$ may carry different dimensional units. Let (again) both eigenstate sets be relabeled as

$$
\begin{array}{cc}
\left|v_{j}\right\rangle \equiv|m\rangle \quad\left|u_{j^{\prime}}\right\rangle=|\theta\rangle \\
\theta=\theta_{0} \epsilon^{2} j^{\prime} \quad m=m_{0} j .
\end{array}
$$

In the $N \rightarrow \infty$ limit one would have

$$
M=\sum_{m=-\infty}^{\infty} m|m\rangle\left\langle m\left|\quad \Theta=\int_{-\pi}^{\pi} \theta\right| \theta\right\rangle\langle\theta| d \theta .
$$

$\Theta|\theta\rangle=\theta|\theta\rangle, \quad\left\langle\theta^{\prime} \mid \theta\right\rangle=\delta\left(\theta^{\prime}-\theta\right), \quad-\pi \leq \theta^{\prime}, \theta \leq \pi$

$$
M|m\rangle=m|m\rangle,\left\langle m^{\prime} \mid m\right\rangle=\delta_{m^{\prime}, m},-\infty \leq m^{\prime}, m \leq \infty
$$

$$
\langle\theta \mid m\rangle=\frac{1}{\sqrt{2 \pi m_{0} \theta_{0}}} \exp \left(\frac{i \theta m}{m_{0} \theta_{0}}\right) .
$$

The modulo $N$ notation have become meaningless to the $|m\rangle$ states, as this label gets unbounded. In the $|\theta\rangle$ states, however, it takes naturally into account the boundary conditions one good set of angle states must have, i.e.,

$$
|\theta\rangle \equiv|\theta(\bmod 2 \pi)\rangle
$$

and the action of the angle shift operator naturally obeys the boundary condition. But it has to be stressed that (as in the Pegg-Barnett scheme), the range of the variable $\theta$ is confined to $[-\pi, \pi)$ by definition, and cyclicity modulo $2 \pi$ is only matter of notation. The modulo $N$ notation in the discrete has ensured that a ket, for example, $|\theta=3 \pi\rangle$ is merely another name of the ket $|\theta=\pi\rangle$.

Therefore, and maybe surprisingly, the usual results for angle-angular momentum variables are recovered from the same discrete root from which the position-momentum results also emerged. Again, the product $m_{0} \theta_{0}$ must be set to $\hbar$. $\theta_{0}$ is not expected to be a dimensional unit but must be related to how one is measuring the angle.

\section{Phase Space}

A phase space formulation of quantum mechanics must be based on a one to one correspondence between operators and c-number functions of two variables

$$
\hat{O} \rightleftharpoons o(p, q)
$$

One safe way to obtain such a mapping scheme is to look for a complete basis on operator space, for in that case the role of the function $o(p, q)$ is played naturally by the decomposition coefficients.

\section{III.1 Discrete case}

In references $[7,6,8,2,9]$ is extensively discussed that the set of operators

$$
\begin{aligned}
G(m, n)= & \frac{1}{N} \sum_{j, l=-\frac{N-1}{2}}^{\frac{N-1}{2}} U^{j} V^{l} \exp \left(\frac{i \pi j l}{N}\right) \\
& \exp \left[-\frac{2 \pi i}{N}(m j+n l)\right],
\end{aligned}
$$

forms a complete and ortoghonal basis in operator space (for simplicity, we're omitting a term that does not influence the mapping of individual operators but that is crucial when we

\footnotetext{
${ }^{1}$ This point was introduced after a question from Prof. B. V. Carlson at the XXV RTFNB.
} 
look at products). The $U$ 's and $V$ 's are the Schwinger unitary operators presented above.

As a basis, the set (31) can be used to represent all linear operators acting on the given $N$-dimensional state space; this can be accomplished by a direct decomposition

$$
\hat{O}=\sum_{m, n=0}^{N-1} o(m, n) G(m, n),
$$

where the coefficient, $o(m, n)$, that gives rise to the representative of the operator $\hat{O}$ in the discrete phase space[6], is given by

$$
o(m, n)=\frac{1}{N} \operatorname{Tr}\left[G^{\dagger}(m, n) \hat{O}\right]
$$

The phase space representative of the density operator in the discrete approach is also referred to as (discrete) Wigner function $[6,7,2,9]$.

\section{III.2 Cartesian coordinates: continuum limit}

If we start from the discrete space operator basis elements, Eq.(31), and proceed to obtain a continuous limit just as in section 2.1 (the Cartesian case), we get for the basis elements (details on[9]),

$$
G(p, q)=\frac{1}{2 \pi \hbar} \int_{-\infty}^{\infty} d v|q+v / 2\rangle\langle q-v / 2| \exp \left[\frac{i v p}{\hbar}\right],
$$

which is exactly the form of the Weyl-Wigner basis elements $\Delta(p, q)[10]$. It is interesting to see that, as in the state space description, the parameter $\delta$ doesn't affect the final result. It is now trivial matter to prove that the decomposition coefficients behave well in the limit and also go to the WeylWigner coefficients. From this follows that the whole mapping scheme is recovered. One immediately concludes that the discrete Wigner function has the ordinary Wigner function as its continuum limit, in the sense discussed above. Most properties of the usual Wigner function are originally present in the discrete one, and come by as the continuum limit of the later.

\section{III.3 Angular coordinates: continuum limit}

Following on our analogy, we choose now the parameter $\delta$ in the extreme situation $\delta=0$. We expect now to obtain a phase space formalism which is consistent with angular coordinates. We start once more from our discrete operator space basis elements, and similar reasoning to the above (details again on [9]) leads to

$$
G(\alpha, l)=\frac{1}{2 \pi} \int_{-\pi}^{\pi} d \alpha\left|\theta+\frac{\alpha}{2}\right\rangle\left\langle\theta-\frac{\alpha}{2}\right| \exp \left[\frac{i l \alpha}{\hbar}\right],
$$

that is precisely the result of references $[11,12]$.
Again, all properties of the angular Wigner function can be obtained from its discrete counterpart by the limiting process above. It must be stated however that in a lot of cases is easier to work in the discrete rather than in the angular case. Also, some features that are considered conditions for the existence of the Wigner function by $[11,12]$ are derived as properties of it in our scheme.

\section{Conclusions}

The basic result here, both in a state space or in a phase space approach, was to show that the two kinds of canonical variables defined on degrees of freedom with classical counterpart can be obtained from a description of a degree of freedom without classical counterpart. The powerful PeggBarnett approach was seen as a particular case of the generalization of an old Schwinger idea.

It is important to observe that the connection between Cartesian and angular coordinates is ultimately lost only after the $N \rightarrow \infty$ limit is taken. As the physical validity of such a limit can itself be put on perspective [13], we emphasize that the ultimate aim of the present discussion is to try understand (even) better the physical meaning of abstract quantum entities.

\section{Acknowledgement}

MR is supported by CNPq, Brazil and SSLM by CAPES, Brazil.

\section{References}

[1] M. Ruzzi, J. Phys. A 35, 1763 (2002).

[2] M. Ruzzi and D. Galetti, J. Phys. A 35, 4633 (2002).

[3] D.T. Pegg and S.M. Barnett, Phys. Rev. A39, 1665 (1989).

[4] J. Schwinger, Quantum Kinematics and Dynamics, (Benjamim, New York, 1960), Chaps 1,2,3.

[5] A. Bohm, The Rigged Hilbert Space and Quantum Mechanics, (Springer Lecture notes in Physics, Vol. 78, 1978).

[6] D. Galetti and A. F. R. de Toledo Piza, Physica 149A, 267 (1988).

[7] D. Galetti and A. F. R. de Toledo Piza, Physica A186, 513 (1992).

[8] D. Galetti and M. Ruzzi, Phys. A 264, 473 (1999).

[9] M. Ruzzi and D. Galetti, J. Phys. A 33, 1065 (1999).

[10] B. Leaf, J. Math. Phys. 9, 65 (1968).

[11] N. Mukunda, Am. J. Phys. 47, 182 (1979).

[12] J. P. Bizarro, Phys. Rev. A 49, 3255 (1994)

[13] B. Leaf, Found. of Physics 12, 583 (1982). 